\title{
Modelo de Enseñanza de las Técnicas de Programación de Producción, basado en Instrucción Invertida
}

\author{
Jaime A. Arango-Marín, Jaime A. Giraldo-García y Omar D. Castrillón-Gómez \\ Facultad de Ingeniería y Arquitectura, Departamento de Ingeniería Industrial, Universidad Nacional de \\ Colombia, Sede Manizales, Campos La Nubia, Bloque Q $2^{\circ}$ piso. Manizales - Colombia. \\ (e-mail: jaarangom@unal.edu.co)
}

Recibido Sep. 4, 2017; Aceptado Nov. 14, 2018; Versión final Ene. 12, 2018, Publicado Oct. 2018

\begin{abstract}
Resumen
Se presenta y analiza una propuesta de formación invertida para la enseñanza de las técnicas de programación de producción buscando tener mayor interés, colaboración y satisfacción de los estudiantes, que en los cursos magistrales. A los participantes en el curso se les plantea un problema de una planta de producción de tamaño real, donde hay que tomar decisiones del nivel táctico y de nivel operativo. Para la toma de las diferentes decisiones se brinda al grupo los fundamentos de las diferentes herramientas, soportadas en aplicativos de software. Estos incorporan técnicas inteligentes aplicadas a las diferentes etapas de la programación. Se valida la efectividad de las herramientas frente al comportamiento de los indicadores claves del área de producción, pudiendo utilizar esas medidas como criterio para la calificación de los estudiantes. La evaluación cualitativa de las pruebas preliminares sugiere que la aplicación de un modelo de este estilo mejora la motivación, la interacción entre estudiantes y profesores, y el desempeño académico.
\end{abstract}

Palabras clave: instrucción invertida; planeación de producción; programación de producción; simulación

\section{Teaching Model of Production Programming Technics, based in Flipped Classroom}

\begin{abstract}
This paper presents and analyzes a flipped classroom proposal for teaching production programming technics, with the aim of increasing the interest, collaboration and satisfaction of the students in comparison with traditional teaching. A real size production plant problem is presented to the students in order to make decisions on tactical level and operative level. To make the decisions, the concepts linked to the different tools are supplied to the students, with the support of software applications. These incorporate smart technics applied to each step of the planning process. The effectiveness of the tools in the key indicators of production area are validated calculating indicator values that could be used as student grades criteria. The qualitative evaluation of preliminary tests shows that the use of this kind of educational model improves motivation, professor-student interaction and academic performance.
\end{abstract}

Keywords: flipped classroom; production planning; production programming; simulation 


\section{INTRODUCCIÓN}

La enseñanza de las actividades de programación y control de la producción, se enfoca en un modelo jerárquico que diferencia los niveles estratégico, táctico y operativo, basado en tanto en el horizonte de tiempo que abarca la planificación, como en los niveles jerárquicos en los que se toman las decisiones. Los cursos de Programación y Control de la Producción tratan las decisiones de planeación y programación en niveles táctico y operativo. La metodología tradicional enfoca el proceso de enseñanza exponiendo los contenidos en forma magistral, enunciando primero los conceptos, explicando luego los procedimientos de solución y aplicándolos a ejemplos académicos de tamaño reducido, cuya solución sea posible dentro del horario de clase. Ese enfoque tiene limitaciones en el desarrollo de la apropiación de los conceptos por parte de los estudiantes dada su remota conexión con el entorno real de producción. Según diversos investigadores, las lecciones tradicionales, por si solas no son lo más recomendable para conseguir los objetivos de aprendizaje (González-Gómez et al., 2016). La escuela tradicional privilegia la memorización de fórmulas y procedimientos, en detrimento de la creatividad e iniciativa de los educandos. Se queda, además, en un formato limitado a los recursos tradicionales del aula sin aprovechar el potencial de las nuevas tecnologías para avanzar un poco más tanto en la comprensión de los temas como en el entrenamiento en su aplicación a la industria. Frente a esas restricciones, hay diferentes propuestas de innovación en las estrategias de enseñanza aplicadas con relativo éxito en diferentes disciplinas; tales como: el aprendizaje basado en problemas, el uso de recursos tecnológicos y el apoyo de las técnicas de simulación. El aprendizaje basado en problemas ha mostrado mejores resultados que los métodos tradicionales (Latasa et al., 2012), trabajando en el aula alrededor de un problema específico, muchas veces tomado del mundo real. Sobre el uso de la tecnología como complemento para potenciar el trabajo académico y para acercar a los estudiantes a entornos cercanos a la realidad, Fredes et al. (2012) afirman que las tecnologías de información y comunicaciones contribuyen a la transformación de los modelos educativos. Un caso exitoso lo presentan Vargas y Giraldo (2015) quienes desarrollaron un grupo de herramientas computacionales para la programación de producción y operaciones. En cuanto a la simulación, Cistac et al. (2009) comentan que permite modelar situaciones y analizar resultados, motivando a los estudiantes hacia el aprendizaje. La simulación se ha aprovechado en temas sensibles de programación de producción como la secuenciación de tareas, como lo presentan Giraldo et al. (2013).

Además del aprendizaje basado en problemas, hay otra estrategia activa de enseñanza de reciente crecimiento conocida como la instrucción invertida (flipped classroom) que propone acceso a los contenidos teóricos usando tecnologías de información y propicia espacios de discusión y construcción de aprendizaje en el aula. La idea de la instrucción invertida es que los estudiantes accedan en su casa a los contenidos temáticos y vayan al aula de clase a trabajar en las tareas o ejercicios prácticos, aprovechando ese espacio de contacto con sus compañeros y profesores. Se llama invertida porque invierte el esquema tradicional en el que los contenidos se exponen en el aula y los trabajos se hacen en casa. Según diversos autores, este enfoque se adapta a las características de los estudiantes actuales, motiva el interés por aprender, y propicia la interacción entre estudiantes y profesores (Bergmann y Sams, 2012; Lage et al., 2000; Strayer, 2012).

La instrucción invertida ha sido probada por diferentes investigadores como una herramienta efectiva para la difusión de los conocimientos, dado que facilita y mejora el aprendizaje. Según Tune, Sturek y Basile (2013) el modelo es un medio muy eficaz para difundir los conceptos clave a los estudiantes. Mason et al. (2013) muestran que: el aula invertida permite cubrir más contenido; los estudiantes se desempeñan igual o mejor en las evaluaciones que con el método tradicional; se adaptan rápidamente y encuentran el formato satisfactorio y efectivo. González-Gómez et al. (2016) hallaron una diferencia estadísticamente significativa en el promedio de las evaluaciones a favor de los alumnos de la instrucción invertida. Además, los estudiantes tenían una percepción favorable sobre la metodología, notando la capacidad de pausar, retroceder y repasar las clases, así como un mayor aprendizaje individualizado y una mayor disponibilidad de los maestros. Chen et al. (2016) concluyeron que, en estos ambientes, las percepciones de los estudiantes sirven como estrategias de motivación en el proceso de enseñanza-aprendizaje para involucrarlos en actividades tendientes a mejorar sus calificaciones. Missildine et al. (2013) establecen que la combinación de nuevas tecnologías de enseñanza con actividades interactivas en el aula puede resultar en un mejor aprendizaje. El estudio de Gómez et al (2017) demostró que los estudiantes con la metodología inversa tuvieron mejor aprendizaje y mejor percepción del contenido y el método de enseñanza. Baepler et al. (2014) encontraron que, en un ambiente de aprendizaje activo, se reducen las consultas a profesores en un $66 \%$, mientras el aprendizaje es significativamente mejor que en un aula tradicional; y mejoran las percepciones de los estudiantes acerca del entorno de aprendizaje.

La motivación para el trabajo académico se incrementa en los estudiantes cuando los docentes aplican instrucción invertida. Davies et al. (2013) concluyen que una instrucción invertida soportada en herramientas tecnológicas, es eficaz y escalable, facilita el aprendizaje, y aumenta la motivación. Para Chen et al (2014), los estudiantes expresaron satisfacción con el curso, además su asistencia y sus esfuerzos de estudio 
aumentaron. Por su parte, Abeysekera y Dawson (2015) proponen que los enfoques invertidos podrían mejorar la motivación del estudiante y ayudar a manejar la carga cognitiva. De otro lado, Zainuddin y Halili, (2016) afirman que la escuela inversa beneficia a los estudiantes tanto en lo académico como en lo motivacional. Rotellar y Cain (2016) encuentran que la escuela inversa es positiva tanto para los profesores como para los estudiantes que aprecian el mayor compromiso. Thai et al. (2017) observan que el estudio en un entorno de instrucción invertida tiene un efecto positivo sobre las creencias de autoeficacia y la motivación intrínseca de los estudiantes, sugiriendo que esa metodología podría ser una forma prometedora de mejorar el rendimiento de aprendizaje de los estudiantes. Sin embargo, O'Flaherty y Phillips (2015) indican que, aunque hay mucha evidencia indirecta de la mejora del rendimiento académico y la satisfacción de los estudiantes y docentes con el enfoque invertido, aún no hay suficientes pruebas concluyentes de que ese enfoque contribuya a la construcción de aprendizaje y otras habilidades a largo plazo.

En oposición a la crítica de O'Flaherty y Phillips (2015), otros investigadores han encontrado evidencia de que la instrucción invertida potencia en los estudiantes el uso de capacidades como el análisis, el pensamiento crítico y la creatividad. Kong (2014) probó un crecimiento estadísticamente significativo en el dominio del tema por los estudiantes, quienes percibieron la efectividad de los diseños pedagógicos de las aulas digitales en el apoyo al desarrollo de competencias de conocimiento y habilidades de pensamiento crítico. McLaughlin et al. (2014) concluyen que este enfoque merece una consideración cuidadosa por parte de los educadores que buscan mejorar el aprendizaje y los resultados y nivelar a los estudiantes para atender los retos de la era actual. Morton et al. (2017) recomiendan el enfoque del aula invertida para beneficiar la retención cuando se espera que los estudiantes analicen el material de aprendizaje. En el estudio de Baytiyeh, (2017), los estudiantes encontraron ventajas en: aprendizaje auto-regulado, habilidades de resolución de problemas, trabajo en equipo y habilidades de comunicación, disfrute y creatividad. Lee et al. (2017) implementaron instrucción invertida obteniendo mejoras en competencias matemáticas, calidad de las reflexiones y satisfacción de los estudiantes.

\section{METODOLOGÍA}

Se propone un enfoque basado en instrucción invertida con soporte de simulación y apoyo de herramientas informáticas para la solución de casos de escala real que simulan un entorno real de producción, donde los grupos de estudiantes se entrenarán en la toma de decisiones referentes a capacidad, políticas de inventarios, programación detallada, asignación de cargas. Se parte de la hipótesis de que este tipo de metodologías mejoran la motivación de los estudiantes en el tema de estudio, generan mejores resultados de aprendizaje y permiten la expresión de la creatividad y el pensamiento crítico en los estudiantes, gracias a la interacción que se logra entre estudiantes y profesores y el contacto con un objeto de estudio basado en el tipo de problemas de gestión de producción que se encuentran en una empresa real.

Para implementar el modelo de instrucción invertida, se ha diseñado un proceso de enseñanza-aprendizaje, siguiendo los 9 principios propuestos por Kim, et al (2014) para diseñar un entorno de instrucción invertida: 1) Ofrecer a los estudiantes la primera exposición al contenido antes de la clase; 2) Incentivar a los estudiantes a que se preparen para la clase; 2) Conectar las actividades en clase y fuera de la clase; 4) Ofrecer orientación claramente definida y bien estructurada; 5) Dar tiempo suficiente para las tareas; 6) Construir una comunidad de aprendizaje; 7) Utilizar tecnologías familiares y de fácil acceso; 8) Evaluar la comprensión del estudiante; y 9) Entregar retroalimentación rápida y adaptable a trabajos individuales o de grupo.

\section{Preparación: Conformación de los grupos, asignación de temas}

Para entrenar a los estudiantes en la toma de decisiones conjugando diferentes puntos de vista, se conforman grupos de trabajo en los que cada uno de los integrantes asume un rol análogo a los entornos de trabajo de la gestión de producción y operaciones, teniendo cada uno asociado un indicador que representa su función y evalúa los resultados colectivos de acuerdo a su aporte. A cada grupo de trabajo se le asigna una empresa con características diferentes en cuanto a su estrategia comercial, su tamaño y su configuración productiva. Cada grupo de trabajo recibe información sobre: registro histórico de ventas por familia de productos; perspectiva comercial de la empresa; capacidad consolidada de la planta por familia de productos; costos de producción, contratación, despido, manejo de inventarios; y niveles iniciales de personal e inventarios.

\section{Primera fase: Programación en el Nivel táctico}

Se solicita a cada grupo generar el pronóstico de ventas para el horizonte de planeación, así como el plan agregado para ese mismo período de tiempo. En la primera etapa, el grupo de trabajo se enfrenta a las decisiones de elegir y parametrizar el modelo de pronósticos más apropiado para la situación planteada. Se 
ofrece vía digital, la formación en modelos de pronósticos, explicando las herramientas de análisis de series de tiempo, presentando diferentes técnicas de pronósticos de tipo cuantitativo y de tipo cualitativo, con apoyo de textos de referencia en el tema como Hanke y Wichern (2006). Como soporte, se entrega a los grupos un aplicativo de software. En la segunda etapa, se solicita al grupo definir el plan agregado en cuanto a la cantidad de recursos a utilizar en cada periodo durante el horizonte de planificación. En este punto, se presenta la capacitación en planeación agregada, las diferentes estrategias (nivelación, persecución, mixtas) y el planteamiento del modelo de optimización aplicable a este tipo de problemas. Se aportan los referentes bibliográficos pertinentes y se utiliza un aplicativo de software. El plan agregado será la base de la programación en la siguiente fase. Al final de esta fase, cada grupo debe haber tomado decisiones sobre el manejo que se le dará durante el periodo de planificación operativa a cada uno de los escenarios posibles, en cuanto al manejo de la capacidad, previendo el posible uso de horas extras o subcontratación, aplazamiento o anticipo de órdenes, y manejo de inventarios. Esta decisión debe ser consecuente con el manejo que se dará en el nivel operativo a ese tipo de situaciones.

\section{Segunda fase - Nivel operativo}

Para generar el programa maestro, la programación de componentes y órdenes de compra y la secuenciación de tareas; a cada grupo de trabajo se le entrega información sobre: configuración de la planta; capacidad consolidada de cada sección y detallada de cada recurso; diseño de los productos; políticas de inventario; niveles actuales de existencias de cada producto, componente y material; pedidos de los clientes para el periodo de planeación; y pronósticos según el plan agregado. El primer producto que deben generar los grupos de estudiantes es el programa maestro, que indica las cantidades a programar de cada producto en cada periodo. Debe validarse la capacidad de acuerdo al plan agregado, de manera que sí la capacidad es insuficiente, se activen alternativas como subcontratación, horas extras, aplazamiento cancelación o adelanto de pedidos. Se facilita instrucción sobre elaboración de programas maestros con apoyo en textos sobre el tema, y recursos de software para facilitar los cálculos. Cada grupo debe tomar decisiones consistentemente con la política seleccionada en el nivel táctico cuando se planteó el plan agregado.

El segundo producto es la programación de fabricación de componentes y órdenes de compra de los ítems requeridos para cumplir con los pedidos. Para calcularla, se requiere aplicar la programación MRP (Material Requirements Planning) que se basa en el programa maestro y en la información de políticas de inventario para determinar las cantidades y los momentos en los que se debe programar cada componente. Por lo tanto, se ofrece la clase en línea sobre cálculo de MRP y se aporta, además, un aplicativo de software para resolverlo. El tercer producto de esta fase es la programación detallada, incluyendo la asignación de trabajos a los diferentes recursos y la secuencia de procesamiento en cada una de las máquinas 0 secciones de producción. Se deben elegir las reglas de secuenciación que permitan la mejor utilización de la capacidad instalada y aseguren el cumplimiento de los compromisos de entrega con los clientes. Como soporte, se ilustran los algoritmos de secuenciación aplicables a los diferentes entornos, considerando diversas funciones objetivo y condiciones realistas que pueden afectar la disponibilidad y la capacidad de los recursos; y se presenta un aplicativo de software que implementa diferentes algoritmos de secuenciación para facilitar los cálculos y generar la programación detallada.

Cada grupo tomará las decisiones respectivas de manera consensuada, buscando el mejor desempeño integral de su subsistema productivo. Dado que cada integrante del grupo es evaluado por un indicador diferente, la idea es que en el proceso se llegue a madurar una decisión entre múltiples opciones, de tal forma que sea equilibrada en cuanto al desempeño del sistema con respecto a las diferentes variables de interés. Como resultado de la planificación, cada grupo generará los listados ordenados correspondientes a cada uno de los elementos solicitados de la programación tanto general como detallada: programa global de producción por período de tiempo; programación de materiales con cantidades y fechas de producción, despacho y entrega; asignación de cargas por recurso productivo; y secuencia de trabajos a procesar por cada recurso.

\section{Simulación y evaluación}

Las propuestas de solución alimentan el modelo de simulación del desempeño del sistema en cuanto a pedidos, eficiencia de los recursos, tiempos de suministro de los proveedores, etc. En forma opcional, se pueden introducir situaciones como fallas en los sistemas logísticos que impliquen cambios en la planificación y que pongan a prueba la previsión y la capacidad de reacción frente a diferentes situaciones. El resultado es una comparación de lo planeado contra lo ejecutado. Se calculan los diferentes indicadores, que serán el criterio de evaluación del desempeño de cada uno de los grupos. Frente a los resultados, los valores de los indicadores y la información de nuevos pedidos y disponibilidades de los recursos, cada grupo generará una nueva propuesta de planeación para el siguiente periodo. Se repetirá el proceso varias 
veces permitiendo el análisis de las fallas, la reacción oportuna frente a los posibles imprevistos y potenciado el aprendizaje. La calificación de cada estudiante estará ligada a la calidad del indicador de desempeño que obtenga su grupo con respecto a su rol específico en el sistema de producción. Esto propiciará una toma de decisiones consensuada a partir de diferentes puntos de vista y un desempeño armónico del sistema productivo frente a las diferentes variables de interés. Cada quien debe aprender a conciliar su criterio personal frente a una meta común.

En resumen, el modelo conjuga: 1) Un problema de tamaño real a ser resuelto; 2) Lecciones en línea (en video y en plataformas digitales); 3) Herramientas computacionales diseñadas para los cálculos requeridos en cada etapa de la planificación; 4) Interacción entre los grupos de estudiantes para la toma de decisiones dentro de la experiencia didáctica; 5) Interacción entre estudiantes y profesores para la asesoría y solución de dudas durante el proceso; 6) Aprendizaje soportado en la experimentación y en los resultados parciales; 7) Varias iteraciones del ciclo de programación para afianzar la experiencia y propiciar el aprendizaje de los conceptos clave del tema; y 8) Evaluación basada en el desempeño de las soluciones para aproximar la experiencia a la situación real.

\section{RESULTADOS}

Para la implementación del modelo se desarrollan una serie de conjuntos de datos que representan diferentes tipos de procesos productivos que corresponden a combinaciones de las características que se muestran en la tabla 1. Una cuarta posible variable puede ser el sector económico, buscando una experiencia más realista.

Tabla 1: Criterios de generación de casos de prueba

\begin{tabular}{|l|l|l|l|}
\hline \multicolumn{1}{|c|}{ Variable } & \multicolumn{1}{|c|}{ Opción 1 } & \multicolumn{1}{c|}{ Opción 2 } & \multicolumn{1}{c|}{ Opción 3 } \\
\hline Tamaño de empresa & Grande & Mediana & Pequeña \\
\hline Sistema & En Línea (Continua, flow shop) & Por proceso (job shop) & Por lotes \\
\hline Programación & Por pedidos & Para inventario & Ensamble bajo pedido \\
\hline
\end{tabular}

Las combinaciones de las 3 opciones de las 3 variables permiten generar $3^{3}=27$ casos diferentes. Según el tamaño de las cohortes de estudiantes (en el caso de la institución donde se está implementando varía entre 30 y 60), sólo se requieren entre 8 y 15 casos, e incluso menos, tomando la opción de tener dos o más grupos de trabajo con cada configuración para tener la oportunidad de hacer comparación directa de los resultados entre diferentes grupos con diferentes criterios en la toma de decisiones frente a una misma situación planteada. Para cada caso, se genera un conjunto de datos de entrada que incluye los elementos que se muestran en la tabla 2. Los datos son generados por simulación Montecarlo, de forma que cada variable se mueva dentro de un rango coherente de valores basados, en lo posible, en entornos reales de producción.

Tabla 2: Información a generar para cada caso de prueba.

\begin{tabular}{|l|l|l|}
\hline \multirow{4}{*}{ Fase } & \multicolumn{1}{|c|}{ Concepto } & \multicolumn{1}{c|}{ Variables } \\
\hline \multirow{4}{*}{1} & Estadísticas de ventas & Familia de productos, período, ventas (Entre 24 y 50 períodos atrás) \\
\cline { 2 - 3 } & Perspectiva comercial & $\begin{array}{l}\text { Percepción del futuro de las ventas hacia el mediano plazo: Crecimiento, } \\
\text { contracción o estabilidad de la economía, el mercado y la participación de la } \\
\text { empresa. }\end{array}$ \\
\cline { 2 - 4 } & Capacidad consolidada & Familia de productos, unidades, tasa de producción por recurso. \\
\cline { 2 - 4 } & Costos. & $\begin{array}{l}\text { Costo unitario producción, costo unitario almacenamiento, costo fijo, costo hora } \\
\text { extra, costo subcontratación, costo venta pérdida, costo contratación y despido }\end{array}$ \\
\cline { 2 - 4 } & Situación Inicial & $\begin{array}{l}\text { Personal inicial, inventario inicial de producto terminado, producto en proceso, } \\
\text { materias primas (Global y en detalle) }\end{array}$ \\
\hline \multirow{4}{*}{2} & Productos & $\begin{array}{l}\text { Código, descripción, materiales, ruta de proceso, tasa de producción por producto } \\
\text { por recurso }\end{array}$ \\
\cline { 2 - 4 } & Capacidad detallada & Producto, recurso, unidades máximas por periodo \\
\cline { 2 - 4 } & Gestión de inventario & $\begin{array}{l}\text { Nivel de servicio deseado, tiempos de abastecimiento por material, Puntos de } \\
\text { reorden, stocks de seguridad, lotes económicos de pedido }\end{array}$ \\
\cline { 2 - 3 } & Pedidos & Producto, cantidad, fecha de entrega \\
\hline
\end{tabular}


Se conforman grupos de trabajo de entre 3 y 4 estudiantes. Cada uno de los integrantes corresponde a un perfil diferente de los que se puede encontrar en un entorno real de producción, en especial en lo que tiene que ver con las variables de interés para evaluar el desempeño del sistema productivo. Cada integrante será evaluado individualmente, de acuerdo al valor relativo del indicador respectivo en comparación con los resultados obtenidos por los otros grupos. El estudiante cuyo grupo logre el mejor valor de un indicador entre todos los grupos tendrá la mejor calificación y los otros tendrán calificaciones proporcionalmente menores. Para la asignación de los responsables y los indicadores, se propone una distribución como se muestra en la tabla 3.

Tabla 3: Perfiles de trabajo e indicadores para los integrantes de los grupos de trabajo

\begin{tabular}{|l|l|}
\hline \multicolumn{1}{|c|}{ Rol } & \multicolumn{1}{c|}{ Indicador } \\
\hline Gerencia de Inventarios & Rotación \\
\hline Gestión de costos & Costo total de la operación \\
\hline Entregas & Tardanza \\
\hline Producción & Uso de la capacidad \\
\hline
\end{tabular}

Para los cálculos necesarios en la programación de producción, se entrega a los estudiantes herramientas de software, que comprenden un modelo de generación de pronósticos de ventas por suavización exponencial con optimización de parámetros por programación no lineal que se puede descargar de goo.gl/29097X y un aplicativo que calcula pronósticos, planeación agregada, programación MRP, secuenciación y modelos de inventarios disponible en goo.gl/4a3mz4. Igualmente se aporta un programa de asignación de cargas y secuenciación de tareas: goo.gl/5Wci2n. Con el apoyo de las herramientas, cada grupo toma las decisiones respectivas y presenta sus propuestas de programación para cada nivel. El sistema de simulación genera los valores de desempeño de cada sistema dentro de los rangos preestablecidos y permite el cálculo de indicadores de producción, eficiencia, niveles de inventarios, y costos. Los resultados detallados de cada grupo se presentan a los integrantes como base para el siguiente periodo de planeación. Se corre el modelo entre 3 y 4 periodos, para permitir un mayor aprendizaje por medio de la experimentación y el análisis de los resultados, que se va afianzando con la experiencia. El modelo propuesto se ha configurado de acuerdo a los principios propuestos en Kim et al (2014). La tabla 4 muestra como se ha hecho la implementación de cada uno de los nueve principios de diseño en la propuesta metodológica para la enseñanza de la programación de producción.

Tabla 4: Implementación de los principios de diseño propuestos por Kim et al (2014)

\begin{tabular}{|c|c|}
\hline Principio & Implementación \\
\hline $\begin{array}{l}\text { Ofrecer a los estudiantes la primera } \\
\text { exposición al contenido antes de la clase }\end{array}$ & $\begin{array}{l}\text { Se da acceso a material bibliográfico y software previo a las sesiones } \\
\text { presenciales }\end{array}$ \\
\hline $\begin{array}{l}\text { Incentivar a los estudiantes a que se } \\
\text { preparen para la clase }\end{array}$ & $\begin{array}{l}\text { Al dar a conocer las reglas de juego se informa que se evaluará según } \\
\text { indicadores y que para alcanzarlos es útil el material compartido }\end{array}$ \\
\hline $\begin{array}{l}\text { Conectar las actividades en clase y fuera } \\
\text { de clase }\end{array}$ & $\begin{array}{l}\text { Las lecturas sugeridas sirven de soporte a la solución de los problemas. } \\
\text { Se combina la simulación con controles de lectura en línea } \\
\text { (evaluaciones) }\end{array}$ \\
\hline $\begin{array}{l}\text { Ofrecer orientación claramente definida y } \\
\text { bien estructurada }\end{array}$ & $\begin{array}{l}\text { El docente debe dar soporte durante todo el proceso, sin ocultar ningún } \\
\text { tipo de información relevante. Todas las preguntas se atienden. }\end{array}$ \\
\hline Dar tiempo suficiente para las tareas & $\begin{array}{l}\text { Se toma una semana entre una iteración y la siguiente de tal manera } \\
\text { que se madure la toma de decisiones y la comprensión de los temas }\end{array}$ \\
\hline Construir una comunidad de aprendizaje & $\begin{array}{l}\text { Cada grupo se convierte en una célula de formación que discute y } \\
\text { estudia los temas e interactúa con los contenidos y los otros grupos en } \\
\text { clase. }\end{array}$ \\
\hline Usar tecnologías familiares de fácil acceso & $\begin{array}{l}\text { Se utilizan hojas de cálculo y aplicativos amigables con interfaces } \\
\text { intuitivas. }\end{array}$ \\
\hline Evaluar la comprensión del estudiante & $\begin{array}{l}\text { Se evalúa el desempeño de la solución propuesta según indicadores } \\
\text { objetivos. Se evalúa también la consulta a las fuentes de información. }\end{array}$ \\
\hline $\begin{array}{l}\text { Entregar retroalimentación rápida y } \\
\text { adaptable a trabajos individuales o grupo }\end{array}$ & $\begin{array}{l}\text { El grupo entrega su propuesta de decisión se evalúa en el sistema de } \\
\text { simulación y se comunica el resultado para preparar la siguiente } \\
\text { iteración. }\end{array}$ \\
\hline
\end{tabular}


El modelo está en vía de implementación completa en pregrado y posgrado para los cursos de Programación de producción en programas académicos de Ingeniería Industrial. Las primeras pruebas se realizaron en grupos de especialización en Dirección de Producción y Operaciones, integrados por un número de estudiantes entre 15 y 18. Se conformaron grupos de trabajo que trabajaron todos con el mismo modelo de empresa y con los mismos conjuntos de datos. La capacitación en los conceptos y las técnicas se hizo en forma presencial en el aula de clase, por lo que se redujo el tiempo disponible para el ejercicio práctico. Se lograron hacer 2 iteraciones del proceso de programación con retroalimentación sobre los resultados frente a la simulación de la ejecución de los programas. En comparación con los cursos del mismo contenido dictados por el mismo docente el año anterior, las calificaciones obtenidas por los estudiantes se incrementaron en un $12 \%$ mientras la valoración del curso por parte de los alumnos mejoró un $22 \%$. Durante el proceso, se aplicaron técnicas como: observación participativa y entrevistas a los estudiantes para evaluar su experiencia de aprendizaje. Al final del curso se solicitó a los estudiantes que expresaran sus percepciones con respecto a la metodología empleada.

Tratando de corroborar la hipótesis planteada acerca de los beneficios de motivación, aprendizaje y desarrollo de análisis y pensamiento crítico, mencionados en las fuentes bibliográficas citadas en la introducción, la tabla 5 resume los hallazgos percibidos de la evaluación cualitativa hecha durante la prueba mencionada.

Tabla 5: Resultados cualitativos observados en las pruebas del modelo frente a expectativa según referentes de literatura

\begin{tabular}{|l|l|l|}
\hline \multicolumn{1}{|c|}{ Característica } & \multicolumn{1}{|c|}{ Referente de literatura } & \multicolumn{1}{c|}{ Resultado observado } \\
\hline $\begin{array}{l}\text { Adaptación estudiantes siglo } \\
\text { XXI }\end{array}$ & Bergmann y Sams, (2012) & $\begin{array}{l}\text { Interacción con recursos tecnológicos, } \\
\text { autonomía para buscar más } \\
\text { información }\end{array}$ \\
\hline $\begin{array}{l}\text { Mayor motivación al } \\
\text { aprendizaje }\end{array}$ & $\begin{array}{l}\text { Davies et al. (2013); Abeysekera y } \\
\text { Dawson (2015); Zainuddin y Halili, (2016) }\end{array}$ & $\begin{array}{l}\text { La asistencia y participación en clase } \\
\text { son más altas que con el método } \\
\text { tradicional. }\end{array}$ \\
\hline $\begin{array}{l}\text { Mayor interacción entre } \\
\text { estudiantes }\end{array}$ & Baytiyeh, (2017) & $\begin{array}{l}\text { Se forma un ambiente altamente } \\
\text { colaborativo en torno a los temas }\end{array}$ \\
\hline $\begin{array}{l}\text { Mayor interacción } \\
\text { estudiantes-profesores }\end{array}$ & $\begin{array}{l}\text { González-Gómez et al. (2016); Rotellar y y } \\
\text { Cain (2016) }\end{array}$ & $\begin{array}{l}\text { Se incrementan las consultas y la } \\
\text { participación. }\end{array}$ \\
\hline $\begin{array}{l}\text { Mejores resultados } \\
\text { académicos }\end{array}$ & Mason et al. (2013); Lee et al. (2017) & $\begin{array}{l}\text { Mejor desempeño en evaluación de } \\
\text { competencias básicas del tema. }\end{array}$ \\
\hline Mayor satisfacción individual & O'Flaherty y Phillips (2015) & $\begin{array}{l}\text { Comentarios positivos, } \\
\text { empoderamiento de los estudiantes } \\
\text { sobre el tema. }\end{array}$ \\
\hline
\end{tabular}

\section{CONCLUSIONES}

Del desarrollo de la propuesta metodológica, su aplicación en los cursos de especialización y su evaluación cualitativa, se concluye que: 1) La metodología propuesta busca generar una experiencia para el estudiante, más cercana a la toma de decisiones sobre producción y operaciones, de tal forma que en el aula se puedan comprender los criterios que se manejan en los entornos reales y las dificultades que implica la planeación, programación y control de la producción de los diferentes sistemas productivos. 2) Las herramientas de software aportan un entorno más realista para los estudiantes, y permiten que se potencie la toma de decisiones por encima de las tareas operativas de cálculo. 3) La parametrización de las herramientas de software desarrolladas para esta propuesta permite que cada grupo incorpore sus visiones particulares, así como probar diferentes alternativas buscando mejorar su desempeño. 4) El carácter iterativo de la propuesta busca un aprendizaje colaborativo, progresivo y experimental, ajustando las decisiones cada vez con más elementos de juicio y mejorando la capacidad de reacción y de previsión de perturbaciones. Cada nueva decisión se soporta en el grupo, en la experiencia y en los conceptos aprendidos. 5) El sistema de evaluación individual basado en indicadores del desempeño global del grupo de trabajo genera un espacio colectivo de toma de decisiones conciliando diferentes puntos de vista y es una oportunidad para aprender a negociar y ceder en posiciones individuales en busca de un mejor resultado colectivo. Ayuda a tener una visión global que en los sistemas productivos es necesaria para tener un balance entre los diferentes objetivos de las organizaciones. 6) Las pruebas realizadas han mostrado consistencia con los antecedentes conocidos en publicaciones científicas sobre experiencias similares. 7) En un futuro se debe evaluar cuantitativamente la implementación completa y proponer mejoras al modelo a partir del aprendizaje obtenido de su aplicación. 


\section{AGRADECIMIENTOS}

Los autores reconocen el apoyo de la Universidad Nacional de Colombia - Sede Manizales, para el desarrollo de esta investigación y expresan su gratitud por el apoyo recibido y por el interés en los resultados de la misma.

\section{REFERENCIAS}

Abeysekera, L. y P. Dawson, Motivation and cognitive load in the flipped classroom: definition, rationale and a call for research, doi: 10.1080/07294360.2014.934336, Higher Education Research \& Development, 34(1), 1-14 (2015)

Baepler, P., J.D. Walker y M. Driessen, It's not about seat time: Blending, flipping, and efficiency in active learning classrooms, doi: 10.1016/j.compedu.2014.06.006, Computers y Education, 78, 227-236 (2014)

Baytiyeh, H., The flipped classroom model: when technology enhances professional skills, doi: 10.1108/IJILT-07-20160025, International Journal of Information and Learning Technology, 34(1), 51-62 (2017)

Bergmann, J. y A. Sams, Flip your classroom: Reach every student in every class every day, International Society for Technology in Education (2012)

Chen, S.-C., S.J.H. Yang y C.-C Hsiao, Exploring student perceptions, learning outcome and gender differences in a flipped mathematics course, doi: 10.1111/bjet.12278, British J. of Educational Technology, 47(6), 1096-1112 (2016)

Chen, Y., Y. Wang, Kinshuk y N.-S. Chen, Is FLIP enough? Or should we use the FLIPPED model instead? doi: 10.1016/j.compedu.2014.07.004, Computers \& Education, 79, 16-27 (2014)

Cistac, G.I., R.H. Bongianino, J.L. Filippi y F.D. Kovac, La Simulación como Medio de Interrelación entre Herramientas Matemáticas y Procesos Tecnológicos, doi: 10.4067/S0718-50062009000500002, Formación Universitaria, 2(5), 3-12 (2009)

Davies, R.S., D.L. Dean y N. Ball, Flipping the classroom and instructional technology integration in a college-level information systems spreadsheet course, doi: 10.1007/s11423-013-9305-6, Educational Technology Research and Development, 61(4), 563-580 (2013)

Fredes, C.A., J.P. Hernández y D.A. Díaz, Potencial y Problemas de la Simulación en Ambientes Virtuales para el Aprendizaje, doi: 10.4067/S0718-50062012000100006, Formación Universitaria, 5(1), 45-56 (2012)

Giraldo, J. A., C. A. Toro y F. A. Jaramillo, Aprendiendo sobre la Secuenciación de Trabajos en un Job Shop mediante el Uso de Simulación, doi: 10.4067/S0718-50062013000400004, Formación Universitaria, 6(4), 27-38 (2013)

Gómez, G., D. González Gómez y otros tres autores, La enseñanza de contenidos científicos a través de un modelo «Flipped»: Propuesta de instrucción para estudiantes del Grado de Educación Primaria, doi: 10.5565/rev/ensciencias.2233, Enseñanza de Las Ciencias, 35(2), 71-87 (2017)

González-Gómez, D., J. S. Jeong y D. A. Rodríguez, Performance and Perception in the Flipped Learning Model : An Initial Approach to Evaluate the Effectiveness of a New Teaching Methodology in a General Science Classroom, doi: 10.1007/s10956-016-9605-9, Journal of Science Education and Technology, 25(3), 450-459 (2016)

Hanke, J. E. y D. W. Wichern, Pronósticos en los Negocios, Pearson Educación (2006)

Kim, M.K., M. Kim, O. Khera, y J. Getman, The Experience of Three Flipped Classrooms in an Urban University: An Exploration of Design Principles, doi: 10.1016/j.ineduc.2014.04.003, The Internet and Higher Education, 22, 37-50 (2014)

Kong, S.C., Developing information literacy and critical thinking skills through domain knowledge learning in digital classrooms: An experience of practicing flipped classroom strategy, doi: 10.1016/j.compedu.2014.05.009, Computers y Education, 78, 160-173 (2014)

Lage, M.J., G. J. Platt y M. Treglia, Inverting the Classroom: A Gateway to Creating an Inclusive Learning Environment, doi: 10.2307/1183338, The Journal of Economic Education, 31(1), 30 (2000)

Latasa, I., P. Lozano y N. Ocerinjauregi, Aprendizaje basado en problemas en currículos tradicionales: Beneficios e inconvenientes, Formación Universitaria, 5(5), 15-26 (2012)

Lee, J., C. Lim y H. Kim, Development of an instructional design model for flipped learning in higher education, doi: 10.1007/s11423-016-9502-1, Educational Technology Research and Development, 65(2), 427-453 (2017)

Mason, G.S., T.R. Shuman y K.E. Cook, Comparing the Effectiveness of an Inverted Classroom to a Traditional Classroom in an Upper - Division Engineering Course, doi: 10.1109/TE.2013.2249066, IEEE TRANSACTIONS ON EDUCATION, 56(4) (2013)

McLaughlin, J.E., M.T. Roth y otros seis autores, The Flipped Classroom, doi: 10.1097/ACM.0000000000000086, Academic Medicine, 89(2), 236-243 (2014)

Missildine, K., R. Fountain, L. Summers y K. Gosselin, Flipping the Classroom to Improve Student Performance and Satisfaction, doi: 10.3928/01484834-20130919-03, Journal of Nursing Education, 52(10), 597-599 (2013)

Morton, D.A. y J.M. Colbert-Getz, Measuring the impact of the flipped anatomy classroom: The importance of categorizing an assessment by Bloom's taxonomy, doi: 10.1002/ase.1635, Anatomical Sciences Education, 10(2), 170$175(2017)$ 
O'Flaherty, J. y C. Phillips, The use of flipped classrooms in higher education: A scoping review, doi: 10.1016/j.iheduc.2015.02.002, Internet and Higher Education (2015)

Rotellar, C. y J. Cain, Research, Perspectives, and Recommendations on Implementing the Flipped Classroom, doi: 10.5688/ajpe80234, American Journal of Pharmaceutical Education, 80(2), 34 (2016)

Strayer, J.F., How learning in an inverted classroom influences cooperation, innovation and task orientation, doi: 10.1007/s10984-012-9108-4, Learning Environments Research, 15(2), 171-193 (2012)

Thai, N. T. T., B. De Wever y M. Valcke, The impact of a flipped classroom design on learning performance in higher education: Looking for the best "blend" of lectures and guiding questions with feedback, doi: 10.1016/j.compedu.2017.01.003, Computers y Education, 107, 113-126 (2017)

Tune, J.D., M. Sturek y D. P. Basile, Flipped classroom model improves graduate student performance in cardiovascular, respiratory, and renal physiology, Advances in Physiology Education, 37(4) (2013)

Vargas, J.M. y J. A. Giraldo, Modelo Didáctico en Toma de Decisiones relacionadas con la Gestión de Producción y Operaciones: Aplicación en Ingeniería Química, doi: 10.4067/S0718-50062015000600011, Formación Universitaria, 8(6), 85-94 (2015)

Zainuddin, Z. y S. H. Halili, Flipped Classroom Research and Trends from Different Fields of Study, The International Review of Research in Open and Distributed Learning, 17(3) (2016) 
